Mots. Les langages du politique

$111 \mid 2016$

Normes et usages de la langue en politique

\title{
L'accent : un marqueur de l'homme politique dans les films de fiction français (années trente) ?
}

El acento: ¿ un marcador del hombre politico en las películas francesas de ficción (años treinta)?

The accent: a marker for politicians in French fiction movies (in the 1930's)?

\section{François Amy de la Bretèque}

\section{OpenEdition}

Journals

Édition électronique

URL : https://journals.openedition.org/mots/22354

DOI : $10.4000 /$ mots. 22354

ISBN : 978-2-84788-836-2

ISSN : 1960-6001

Éditeur

ENS Éditions

\section{Édition imprimée}

Date de publication : 10 septembre 2016

Pagination : 63-80

ISBN : 978-2-84788-835-5

ISSN : 0243-6450

\section{Référence électronique}

François Amy de la Bretèque, «L'accent: un marqueur de l'homme politique dans les films de fiction

français (années trente) ? », Mots. Les langages du politique [En ligne], 111 | 2016, mis en ligne le 10 septembre 2018, consulté le 23 avril 2022. URL : http://journals.openedition.org/mots/22354 ; DOI : https://doi.org/10.4000/mots.22354 


\section{L'accent : un marqueur de l'homme politique dans les films de fiction français (années trente)?}

Le film d'actualité, désormais sonore et parlant dans les années trente, a laissé de nombreux témoignages de la parole et de l'accent des hommes politiques dans les circonstances de leurvie publique. Il représente un matériau de premier ordre pour les linguistes et politologues, à condition que l'on prenne en compte les contraintes spécifiques de la diffusion de la parole et de l'enregistrement cinématographique ${ }^{1}$.

Mais ce ne sera pas mon objet. On préférera dans cette étude s'adresser au film dit «de fiction». Celle-ci, disent Jacques Aumont et Michel Marie (2001), « est une forme de discours qui fait référence à des personnages ou à des actions qui n'existent que dans l'imagination de leur auteur et par la suite dans celle du lecteur/spectateur» (c'est la définition commune) ; ils ajoutent : «ce discours [...] n'est en principe pas à prendre au sérieux : il n'engage pas celui qui le profère comme un jugement ou une proposition du langage de la vie réelle. Il relève du processus de "fictivisation" (Odin, 2000) [...] L'acte d'énonciation n'assume pas les engagements qui sont alors normalement requis par cet acte (Searle, 1982) ». Néanmoins, chacun sait bien que derrière le discours des personnages de fiction, l' «énonciateur» (pour faire vite, on dira : le réalisateur et son équipe) se fait le porte-voix d'une opinion, d'une idéologie qui l'englobe.

Le choix de demander à ses interprètes de prendre un accent ou de n'en pas prendre exprime des stéréotypes ou des "prototypes», "des connaissances partagées qui [...] sont utiles comme points de repère pour toute évaluation, pour faire des prédictions, pour organiser notre perception » (Woehrling, 2008, dans Boula de Mareüil et al., p.151).

On se demandera d'abord si l'arrivée du parlant a signifié l'irruption des accents dans le cinéma français, comme on le dit souvent, dans le cas particulier des hommes politiques comme personnages de fiction. On étudiera ensuite si ces accents, ou leur absence, supportent une «évaluation » de ces hommes et de leur fonction, et notamment du rapport qu'ils entretiennent avec

1. Les orateurs à cette époque parlent sans micro; d'autre part, les micros d'enregistrement de ce temps sont non directionnels et la bande passante du film à son optique est très réduite. 
le territoire qui les a élus. L'analyse devra mettre en correspondance les signes linguistiques du dialogue avec les éléments de l’image et de la mise en scène.

\section{Des représentations de l'homme politique étonnamment peu nombreuses}

Le «cinéma du sam'di soir», comme on a appelé le cinéma populaire français des années trente, foisonne de représentations de la politique, du moins dans le souvenir que l'on en a. Car recension faite, la représentation directe des élus et représentants du peuple de la Troisième République y est relativement peu abondante 2 . C'est soit par incidence dans des intrigues où il n'occupe pas le premier plan, soit par déplacement dans des lieux imaginaires, que l'homme politique s'incarne sur les écrans français.

Pierre Billard et Jean-Pierre Jeancolas, deux des meilleurs historiens de la période, les ont inscrits sous la rubrique de l'antiparlementarisme : "Le roman des tricheurs », titre le premier (Billard, 1995, p. 220-228), tandis que le second parle carrément de «films de droite » (Jeancolas, 2005, p. 128). Tous ces films, ou quasiment tous, dérivent du théâtre de boulevard et sont l'adaptation de pièces à succès, ce qui fait dire à Billard que c'est « du cinéma de chansonniers » (ibid.).

Un petit corpus peut donc être constitué, que voici3 :

- Aux urnes citoyens (Jean Hémard, 1932, avec Henri Poupon et Jean Bélières) (PP 11 mars 1932);

- Topaze, de Louis Gasnier, 1933, avec Jouvet et Pauley (PP début janvier 1933);

- Le Père Lampion, de Christian-Jaque, 1934, avec Tramel (PP 21 décembre 1934);

- Le Roi, de Pierre Colombier, 1936, avec Raimu (PP 30 octobre 1936);

- Hercule, d'Alexandre Esway, 1937, avec Fernandel (PP 4 mars 1938);

- Les Hommes nouveaux, de Marcel L'Herbier, 1936, avec Harry Baur (PP =?);

- Eusèbe député, d’André Berthomieu, 1938, avec Michel Simon (PP 9 mars 1939).

Si limité qu'il soit, et compte tenu du fait que certains de ces films demeurent inaccessibles aujourd'hui, il n'en demeure pas moins significatif. Dans cette étude, je m'attacherai plus particulièrement à deux d'entre eux, Topaze et Le Roi. Il faut faire un sort rapide aux autres. On s'apercevra tout de suite du caractère répétitif des situations et des intrigues qui ne mettent en jeu qu'un nombre limité de ressorts simples.

Aux urnes, citoyens! se situe à "Orgepré sur Clèves », autrement dit n’importe où en France profonde. Selon le résumé de Chirat (1975, nº 94), « un riche

2. Pour s'en convaincre, voir Chirat (1975).

3. En matière de cinéma, il est toujours difficile d'affirmer catégoriquement l'exhaustivité d'un corpus. 
industriel nommé Dupont-Lebaigne (Léon Bélières) ambitionne la députation. Suivent intrigues et combines habituelles; Il dépense des sommes considérables et n'est pas élu. Mais le mariage de sa fille (Rosine Deréan) qui trouve le bonheur avec un journaliste qui avait refusé de parler contre lui (Claude Dauphin) le console de son échec $» 4$.

Le Père Lampion est un film de Christian Jaque sur un scénario de René Pujol, d'après une pièce de Jean Kolb et Léon Bélières. Il se déroule dans une république imaginaire, la Carvolie. Un président du Conseil corrompu est enlevé par des comploteurs; un égoutier, qui lui ressemble comme deux gouttes d'eau, lui est substitué. On le chapitre et on essaie de l'utiliser, mais il ne joue pas le jeu car il est honnête. Il se prend au rôle et il réussit à restaurer la confiance. À la fin du film il se retire au profit de Desnoyaux, le vrai président du Conseil, qui a compris et s'est amendé (Jeancolas, 2005, p.122-123)5. Il a été commenté à l'époque par Rémy Pithon, qui l'a rapproché des affaires politiques du moment : le retour au pouvoir de Gaston Doumergue dans l'été 1934, son appel à un exécutiffort, les scandales qui entouraient André Tardieu, la xénophobie rampante (Pithon, 1977; Amy de la Bretèque, 1999).

Hercule, scénario et dialogues de Carlo Rim, réalisé par Alexandre Esway, commence dans un port de pêcheurs du Midi de la France. Hercule (Fernandel) est prud'homme au tribunal de pêche. À ce poste, il fait preuve d'honnêteté et de modération. Voilà qu'il hérite d'un journal parisien à gros tirage, L'Incorruptible (on notera bien sûr ce nom robespierriste!), que possédait son demi-frère dont l'existence lui était demeurée cachée jusqu'à la mort de son père (Édouard Delmont). Le décès de ce demi-frère en fait de facto le propriétaire. "Le rédacteur en chef, Vasco (Jules Berry), profite de son ignorance pour tripoter sans danger. Mais Hercule se rend compte petit à petit du rôle qu'on lui fait jouer et, aidé par sa secrétaire (Gaby Morlay), il renvoie Vasco, donne son journal à "ceux qui le font” et retourne dans son village » (Chirat, $\left.1975, n^{0} 580\right)^{6}$. On le voit, il s'agit d'une nouvelle variation sur le thème du naïf qu'on aurait dû prendre au sérieux, tandis que le dénouement a un petit air de parenté avec Le Crime de Monsieur Lange (film de Jean Renoir, 1935). Les travaux de cet Hercule qui nettoie les écuries d'Augias de la presse corrompue touchent au thème politique, ne serait-ce que par la fonction symbolique qu'il remplit au début et à la fin du film, mais surtout par les dossiers qu'il aura à démêler, parmi lesquels émerge une affaire de malversation dans l'exploitation du pétrole du Sahara. Pour une fois, l'ancrage y est assez précis et l'accent de Fernandel y tient un rôle. "Vous n'êtes pas parisien, ça se voit », lui dit la secrétaire à son arrivée à Paris : on relèvera le déplacement dans le registre de la perception... Mais en l'espèce, cette notation est un compliment sous-entendant qu'il apporte avec lui la pureté de ses origines provinciales.

4. Ciné-Miroir, $\mathrm{n}^{\circ} 364,25$ mars 1932 : récit du film.

5. Récit du film dans Ciné-Miroir nº 508, 28 décembre 1934.

6. Idem, n⿳0707, 21 octobre 1938. 
Dans Eusèbe député, Eusèbe Bonbonneau (Michel Simon), clerc de notaire de province, est l'objet d'une tentative de manipulation de l'aigrefin Jacassar, propriétaire d'une écurie de course et directeur de théâtre (Jules Berry), qui veut le présenter à la députation comme homme de paille. Il pense utiliser sa naïveté, sa maladresse, son aspect rustaud. La sœur de Bonbonneau (Marguerite Moreno) a beau essayer de l'en dissuader, Eusèbe «monte » à Paris. Jacassar utilise comme appât sa propre maîtresse, « une de ces beautés roumaines trépidantes et à l'accent grasseyant $[\mathrm{sic}]^{7}$ » (Elvire Popesco). Mais Bonbonneau ne tarde pas à se rendre compte des combines dans lesquelles on veut l'entraîner, il se révolte et rentre dans son village. L'action se passe en «Creuse et Cher», à nouveau, donc, n'importe où en France profonde. Mais si l'on excepte le personnage joué par Elvire Popesco, les autres n'ont pas d'accent marqué. Le chroniqueur du Film complet livre juste une notation sur la diction hésitante et brouillée de Michel Simon, particularité sur laquelle l'acteur avait fondé en bonne partie sa popularité.

On aura remarqué la petite comédie humaine que nous livrent ces films mis bout à bout, qu'accentue le fait que les comédiens reviennent d'un film à l'autre en reprenant des emplois attachés à leur personnalité.

\section{La question de l’accent replacée dans l'histoire du cinéma}

La question qui nous occupe étant celle des normes et usages de la langue en politique, il faut tout d'abord tenir compte d'une circonstance essentielle et qui prend valeur de paradigme : nous sommes alors au début du cinéma parlant.

On sait bien que la nouvelle technique introduisait dans le cinéma la diversité des langues et, en principe, celle des langages. Michel Chion écrit : «Dans l'histoire du cinéma parlant français, le langage tient une place particulière, liée à la littérature d'une part, au goût de la langue savoureuse et du mot bien choisi d'autre part» (2003, p. 86). Il souligne que c'est la raison pour laquelle la fonction de dialoguiste a été si longtemps autonome dans l'industrie cinématographique. Michel Chion pense après Henri Langlois que c'est le succès de l'Angèle de Pagnol (1934) qui aurait imposé la présence des accents dans le parlant français, ici sous la forme de l'accent méridional. Celui-ci est à l'origine de toute une production qu'a étudiée Claudette Peyrusse - mais celle-ci, en essayant de recenser les hommes politiques «à accent» dans cette filmographie, se trompe plusieurs fois (Peyrusse, 1986, p. 33, 110) ${ }^{8}$.

Michel Chion ajoute que s'est produit plus tard « un certain dessèchement du cinéma français populaire », après la disparition de dialoguistes vedettes

7. Le Film complet du mardi, $\mathrm{n}^{\circ} 2285,4$ juillet 1939 (pour l'ensemble des citations relatives à ce film).

8. Pour les cas d'Henri Poupon, Berval et Léon Bélières dans Aux urnes citoyens, film qui ne se déroule d'ailleurs pas dans le Midi (p. 93). 
comme Michel Audiard, « disparition coïncidant avec l'abandon de tout accent local» au profit d'une «langue cinématographique» standard, en particulier à l'époque de la Nouvelle vague et de ses épigones (Chion, 2003, p. 86-90). C'est encore le cas aujourd'hui, à quelques réserves près.

Ce dessèchement était déjà présent dans toute une partie - et non la moindre - de la production française des premières années du parlant.

Par ailleurs, ainsi que le souligne également Michel Chion, le parlant n'a pas spontanément et automatiquement de visée naturaliste : «Tout le cinéma parlant ne vise pas au même naturel, ou n'y a pas visé tout de suite; nous sommes aujourd'hui frappés par le caractère déclamatoire de certains premiers films sonores» (2003, p.48). La technique de prise de vues avait sa responsabilité en la matière, mais ceci n'explique pas tout.

Les hommes politiques de notre corpus, sauf exception (Hercule), n'ont pas d'accent régional caractérisé, et se distinguent plutôt par leur élocution : c'est elle qu'il faut donc étudier.

Un détour par quelques points de méthodologie est nécessaire. Au cinéma comme dans tous les arts de la représentation, il faut distinguer l'acteur, le rôle, le personnage (Nacache, 2003, p.90).

Nous parlons ici du langage du personnage, c'est-à-dire de l'entité qui agit dans la fiction, la créature diégétique. Mais il est clair qu'un brouillage peut se produire avec celui de l'acteur, surtout du côté du public qui le reconnaît et en conçoit un certain nombre d'attentes. «Tout acteur connu apporte avec lui un sous-texte composé des personnages qu'il a touchés» (ibid., p. 92). La direction d'acteurs est une composante essentielle de la mise en scène, surtout quand il s'agit de l'adaptation de pièces de théâtre.

En outre, le cinéma de fiction côtoie le cinéma d'actualités et il peut arriver, il arrive même souvent, que l'un déteigne sur l'autre. Mais je laisserai de côté ici la question des ressemblances ainsi que celle de la mise en scène de personnages réels. Un seul exemple servira de spécimen de ce type de brouillage : Les Hommes nouveaux de Marcel L'Herbier (1936), tiré d'un roman de Claude Farrère qui se situe dans le Maroc de 1908 - ce qui en fait aussi un film historique et un film colonial. Le personnage principal est un brasseur d'affaire (joué par Harry Baur) qui est partagé entre la passion de ses entreprises et sa jeune femme. Il choisira de continuer à construire le Maroc nouveau. La politique est bien présente en l'espèce de l'entreprise de colonisation et surtout de ses liens avec la vie politique de la Métropole. Un préambule y montre en effet Lyautey (Gabriel Signoret) et Clemenceau (Gustave Gally) défendant la nécessité de garder à tout prix un certain comptoir dans ce pays, contre le scepticisme des politiques. L'intérêt de ces scènes est de nous livrer l'idée que l'on se faisait (et que l'on se fait encore) de la manière de parler des responsables politiques. On prête à Clemenceau un phrasé sec, bougon, viril en somme, et à Lyautey un parler laconique, militaire : le discours de deux chefs. 
Le dialogue est une composante essentielle d'un film parlant, dont il ne faut pas détacher les éléments supra-segmentaux, comme l'accent, l'élocution, la démarche, la façon de se tenir... qui sont autant de modalités de la communication des personnages entre eux, et qui sont, dans le cas d'une mise en scène cinématographique, l'objet de deux réglages : celui du metteur en scène mais aussi celui de l'acteur.

Le réalisateur assume d'autres fonctions au cinéma que la direction d'acteurs. Il décide du cadre, du mouvement, intervient éventuellement au montage. II ne faut donc pas se contenter d'une simple étude de contenu des dialogues, laquelle courrait le risque de ne rien apporter de plus. L'analyse de films doit prendre en compte les dimensions spécifiques de l'expression cinématographique.

David Smadja a souligné que la tâche est complexe quand «il s’agit de relier le cinéma - appréhendé comme approche spécifique du monde [...] et la science politique, c'est-à-dire de rapprocher la production et/ou la perception d'un agencement d'images en mouvement [...] et la recherche des structures sous-jacentes des phénomènes politiques [...] il apparaît que le cinéma constitue une pensée originale de l'objectivation des situations humaines ». Il invite à «mettre entre parenthèses les approches sociologiques et historiques qui visent à expliquer le cinéma par la société présente ou passée et qui se désintéressent du cinéma en tant que tel [...] pour appréhender le cinéma en lui-même.» (Smadja, 2010, p. 5-7).

\section{Études de cas}

\section{Topaze première version}

\section{L'accent du théâtre et celui du cinéma}

Le film réalisé par Louis Gasnier a été tourné en 1932 et est sorti en janvier 1933. C'est l'adaptation à l'écran de la pièce écrite par Marcel Pagnol, créée au Théâtre des Variétés en octobre 19289. Il s'agit de sa deuxième pièce. La première : Marchands de gloire, au théâtre de la Madeleine en avril 1925, avait remporté un certain succès et lancé sa carrière d'auteur dramatique.

Le succès de la pièce conduit Pagnol à envisager une adaptation à l'écran, car il croit fermement en sa potentielle réussite ; il a déjà défini dans une série d'articles sa conception du cinéma qu'on résume volontiers (et abusivement) par la formule «du théâtre en conserve $»^{10}$.

9. Si la pièce n'a pas d'ancrage méridional, ses trois adaptations cinématographiques mettent quant à elles en jeu la question de l'accent.

10. Ses articles dans Les Cahiers du film, de décembre 1933 à février 1934 - quasi contemporains de l'adaptation de Topaze -, avaient soulevé une controverse fameuse mais qui n'est pas notre propos. 
À cette date, Pagnol ne s'est pas encore risqué derrière la caméra. Les producteurs ne lui font pas confiance (Beylie, 1974; Pompa, 1986). Il y viendra en 1933 avec Jofroi. La réalisation est donc confiée à Louis Gasnier, un vétéran de la maison Pathé, célèbre pour avoir réalisé aux États-Unis en 1914-1915, pour le compte de la filiale américaine de la Maison au Coq, les fameux serials connus en France sous le titre Les Mystères de New York. Le fait que le réalisateur ait été formé au cinéma muet n'est pas sans importance et se sent dans sa mise en scène.

Pour éclairer la position de Pagnol par rapport au sujet politique, il n'est pas inutile de commencer en rappelant ce qu'il dit de ses intentions d'alors, $\mathrm{si}$ l'on en croit la Préface bien postérieure (1964) qu'il donna à la réédition de la pièce dans ses CFuvres complètes.

Il raconte qu'avec son voisin et conseiller «Jacques», ils ne lisaient que la page théâtrale de AuxÉcoutes ou Comoedia, «car la politique ne nous intéressait d'aucune façon. Le changement de direction d'un théâtre nous paraissait beaucoup plus important que les changements de ministère, qui étaient d'ailleurs plus fréquents. Je n'aurais certainement pas remarqué la chute du ministère Herriot ${ }^{11}$, si cette catastrophe politique n'avait empêché le Président ou le ministre d'assister à la générale des Marchands de gloire» (Pagnol, 1964, p. 283). Il n'y revient jamais par la suite, se contentant d'une allusion au scandale de Panama dont il avait entendu parler dans son enfance (ibid., p. 286). Cet apolitisme affiché ne trompe évidemment personne. Il est passible du jugement de Pierre Bourdieu (1982, p.154), pour qui celui qui s'affirme apolitique cache ses options politiques de droite.

Mais pour nous, ce qui est utile est le parallèle établi par Pagnol entre le monde du théâtre et celui de la politique, lieu commun, certes, mais que les films du début du parlant réactivent.

Le personnage principal de la pièce, et du film, c'est l'instituteur Topaze, qui, renvoyé de son établissement (la pension Muche), est engagé par un homme politique pour devenir son prête-nom comme agent d'affaires; au départ scandalisé, il se pique au jeu et finit par évincer son "protecteur». C'est, comme le dit Marcel Pagnol, « un homme pur entraîné - sans rien y comprendre - dans de louches combinaisons», lequel, «lorsqu'il découvr[e] avec une grande amertume la toute-puissance de l'argent, se révolt[e]; puis, déjà contaminé lui-même, il accept[e] les nouvelles règles du jeu » (Pagnol, 1995, p. 288). Le destin de Topaze doit être lu au second degré.

Mais la charge politique de la pièce est portée pour l'essentiel par le personnage qui propulse Topaze dans les affaires louches : le conseiller municipal Castel-Bénac. Second grand rôle de la pièce, tout l'acte II et une grande partie

11. Édouard Herriot fut président du Conseil et ministre des Affaires étrangères du 14 juin 1924 au 10 avril 1925. C'était l'époque du fameux « cartel des Gauches». 
des deux actes suivants lui sont consacrés. Il se livre à des prévarications en faisant voter des équipements dont il contrôlera le marché et qui lui permettront de toucher des commissions : la fameuse affaire des balayeuses municipales (II, 1, Pagnol, 1995, p. 378); il acquiert des immeubles qu'il revend à la ville pour construire une rue (II, 11, p.409); il se fait verser des pots-de-vin pour faire voter des décisions, comme le montre l'histoire de l'urinoir mobile, dont les propriétaires de brasserie veulent se débarrasser tour à tour (III, 2, p. 420)12.

Les entreprises coloniales sont un autre secteur d'activité de Castel-Bénac : l'affaire des "porcheries du Maroc » a envoyé un de ses collaborateurs en prison, la vente de terres de Madagascar à ceux qui les habitent, un autre (II, 15, p. 389). Castel-Bénac a également acheté des terres au Maroc (IV, 1, p.440) par un intermédiaire, sa maîtresse Suzy, qui lui réclame une commission (on retrouve ce thème dans plusieurs films des années trente, on le verra dans Le Roi). Signalons que si ces allusions figurent dans les deux premières adaptations, elles feront l'objet de coupes demandées par la censure dans la version de 1950 (voir Sadoul, 1951).

Précisément, c'est pour remplacer son homme de paille que Castel-Bénac recrute Topaze (II, 6, p.392) en remplacement de Roger de Berville, qui tenait jusque-là ce rôle et dont les exigences financières sont devenues trop élevées.

En ce qui concerne l'ancrage, le texte de la pièce comporte une indication aussi vague que possible : «l'action se passe de nos jours ${ }^{13}$ dans une grande ville» (p.334). Les didascalies ne donnent jamais de précision et aucune indication sur l'accent ou la diction des personnages, si ce n'est que Topaze «parle fort ». Liberté est donc laissée aux interprètes et à leur metteur en scène. Dans les films, ce non-ancrage rejoint une raison intrinsèque au cinéma français, le centralisme, matérialisé par l'abandon de tout accent local au profit d'une langue standard, mais il n'était pas fatal et fait aussi sens à l'intérieur du propos du film ${ }^{14}$.

\section{Trois Topaze, des films à ancrage plus ou moins marqué}

Il faut évoquer ici le cas particulier représenté par Topaze, qui connut trois adaptations dans le cinéma français. Je m'arrêterai sur la première, mais la comparaison des trois versions et de leurs divers interprètes serait très instructive et il faut en dire un mot.

Dans la première adaptation cinématographique (1933), c'est Louis Jouvet qui tient le rôle. Marcel Pagnol avait pensé à lui pour la création de la pièce

12. Pierre Chenal s'en resservira dans son adaptation du Clochemerle de Gabriel Chevalier en 1947.

13. Ce qui paraît curieux, car déjà en 1928, l'école de Topaze est anachronique : d'ailleurs, les critiques du film de 1933 soulignent l'aspect décalé du personnage. Ciné-Miroir parle par exemple de son «air épouvantablement décalé» ( $n^{\circ} 379$, 3 juillet 1932).

14. Dans les médias, la radio en particulier, comme au théâtre, elle est aussi déterminée par la formation dans les conservatoires et les écoles. Les témoignages sont nombreux d'acteurs auxquels on a demandé de gommer leur accent. 
(Pagnol, 1995, p. 297, 301, 304). C'est son premier rôle au cinéma. Nous ferons plus loin un sort à sa diction et à son jeu, mais disons tout de suite qu'il crée un personnage «non situé» géographiquement, comme l'est cette première adaptation tout entière. Topaze est tourné dans les studios de Joinville. Les gamins qui figurent les élèves de l'école au premier acte ont été recrutés dans le quartier.

La deuxième version, celle de 1936 mise en scène par Marcel Pagnol luimême - et que le réalisateur a ensuite reniée, au point de la faire disparaître des circuits -, «méridionalise» Topaze, du moins au niveau de la distribution. Le personnage est interprété par Alexandre Arnaudy, un acteur né et mort à Marseille et qui a fait toute sa carrière dans des rôles méridionaux. Néanmoins, les quelques critiques d'époque ne signalent pas qu'il ait affecté le moindre accent.

La troisième version, celle de 1950 que tout le monde connaît, est interprétée par Fernandel. Mais c'est un Fernandel sans accent du Midi. Georges Sadoul lui-même écrivit à la sortie du film : «... Personne ne fut trompé par cette précaution oratoire [de la didascalie de la pièce qui reste dans le vague, voir cidessus] : Topaze se passait à Paris et l'on appela désormais des topazes les conseillers municipaux affairistes» (Sadoul, 1951).

Face à Topaze, il y eut aussi trois Castel-Bénac successifs. On doit noter au préalable la consonance clairement méridionale du patronyme du personnage (inspiré de noms réels, comme Castil-Blaze?) qui connote une origine provinciale.

En 1933, face à Louis Jouvet, c’est Paul Pauley qui donne la réplique. La rondeur, son trait définitoire, face à l'angulosité. Pauley était alors un acteur de vaudeville que Marcel Pagnol se proposait de transformer en acteur de comédie.

En 1936, face à Alexandre Arnaudy, on trouve Léon Bélières. Acteur parisien par excellence, il est né et mort dans la capitale, a fréquenté le Conservatoire et a débuté sur les planches en 1904. Porte Saint-Martin, Bouffes Parisiens; c'est un acteur «de boulevard ». Tôt passé au cinéma avec Max Linder (1911), il a une filmographie très abondante et s'est spécialisé peu à peu dans des rôles de directeur, de curé, d'aristocrate, de mari cocu... Il est et reste un des «seconds rôles» notoires du cinéma français.

En 1950, Jacques Morel donne la réplique à Fernandel. Beaucoup plus mince que les précédents, il est aussi plus jeune : il a débuté dans le cinéma en 1944 et fera une seconde carrière à la télévision. Sa filmographie compte surtout des films populaires.

Donc, aucun des Castel-Bénac n'est caractérisé par l'accent du Midi, alors que cet accent était mieux admis que d'autres dans ces fonctions ${ }^{15}$. L'homme

15. Philippe Boula de Mareüil déclarait récemment : «L'accent méridional, quant à lui, qui évoque le soleil et les vacances, trône au sommet d'une hiérarchie de “français enchantés”, selon l'expression employée par le sociolinguiste Médéric Gasquet-Cyrus»; mais il rappelait plus haut 
politique, ici du moins, n'a pas d'accent lui servant de marqueur. Il faut chercher ailleurs sa caractérisation.

\section{Le diagramme des personnages dans Topaze 1933 : comment les personnages se situent dans l'émission orale de la langue française}

Si grande que soit la part de la langue qui échappe à la variation, il existe, dans l'ordre de la prononciation, du lexique et même de la grammaire, tout un ensemble de différences significativement associées à des différences sociales qui, négligeables aux yeux du linguiste, sont pertinentes du point de vue du sociologue parce qu'elles entrent dans un système d'oppositions linguistiques qui est la retraduction d'un système de différences sociales.

Les usages sociaux de la langue doivent leur valeur proprement sociale au fait qu'ils tendent à s'organiser en systèmes de différences (entre les variantes prosodiques et articulatoires ou lexicographiques et syntaxiques) reproduisant, dans l'ordre symbolique des écarts différentiels, le système des différences sociales. (Bourdieu, 1982, p. 41)

De fait, dans le petit univers de la fiction, les personnages de Topaze se distribuent sur les pôles d'un diagramme relativement clair dont les typologies de prononciation servent de marqueur. Or, ce n'est pas en soi que cette prononciation est porteuse d'axiologie, mais c'est en entrant dans un système. "La valeur naît toujours de l'écart, électif ou non, par rapport à l'usage le plus répandu. Des usages de la langue comme des styles de vie, il n'est de définition que relationnelle», dit encore Bourdieu. Et d'énumérer : "le langage "recherché”, "choisi”, “noble”, "relevé”, "châtié”, "soutenu”, “distingué” ", qui «enferme une référence négative [...] au langage "commun”, "courant”, “ordinaire”, "parlé” ». Au-delà on trouve le langage «"populaire”, “cru”, "grossier”, "relâché”, “libre”, "trivial”, “vulgaire” " (Bourdieu, ibid., p. 50-51).

Bourdieu définit deux critères de jugement qui organisent ces écarts : "le degré de contrôle qu'ils [les parlers] manifestent et l'intensité de la correction qu'ils supposent».

Représentant du langage soutenu, le personnage de Roger de Berville est introduit à la $27^{\mathrm{e}}$ minute dans le salon privé de l'élu Castel-Bénac pour discuter de l'entreprise des balayeuses municipales. Suzy, la maîtresse du conseiller municipal, est présente et participe à la conversation. L'appartenance de classe chez de Berville est assumée et revendiquée. À Suzy, qui dit en aparté : «la particule a sa valeur», il rétorque : «on ne peut nier qu'elle ne soit supérieure au trait d'union [celui de Castel-Bénac] » (II, 4 dans la pièce, 27' 40 dans le film). L'élocution très contrôlée, l'émission marquée par aucun accent de ter-

l'opinion de Jacques Derrida : «L'accent, quelque accent français que ce soit, et avant tout le fort accent méridional, me paraît incompatible avec la dignité d'une parole publique». («Les accents participent à la richesse de notre langue », Le Monde du samedi 27 décembre 2014). 
roir, il pratique une langue faussement naturelle, légèrement marquée comme «snob» et connotée négativement comme telle.

Castel-Bénac, l'homme au trait d'union, use d'un langage maîtrisé, cet autocontrôle résultant d'un apprentissage, comme le montre le discours qu'il ressert dans la conversation (il l'avait fait au conseil municipal et donc appris par cœur), mais chez lui, le naturel revient parfois au galop. Il reste « peuple ». Il lui arrive de lâcher des grossièretés, des familiarités («foutez le camp!»). Mais plus encore que le lexique, c'est sa tenue corporelle et sa tonalité d'émission qui le trahissent. Son bagout, sa gouaille, sa gestuelle excessive, la rapidité de son émission (il élide fortement tous les «e») trahissent sa vulgarité. Sa maîtresse Suzy l'en accuse ailleurs (71', et dans la pièce : IV, 2), tout comme Roger l'en avait critiqué par sous-entendus («Monsieur, dans votre famille, on fout le camp, dans la mienne, on prend congé »). Il essaie vainement de rester au niveau de sa fonction («je suis un élu du peuple, je n’ai pas le droit de me laisser insulter $»)$.

Suzy, sa maîtresse, interprétée par Elvire Popesco (que nous retrouverons affectée d'un accent «d'origine» dans Le Roi) parle ici un français «pur». On ignore son origine, qu'on peut supposer plus basse que la position où elle est parvenue. Elle surjoue l'aisance face à Roger de Berville, tout comme elle surcorrige sa langue et sa prononciation : c'est d'autant plus vrai en l'espèce qu'Elvire Popesco était roumaine.

Topaze, introduit dans la scène suivante (II, 6; film : 32' 40 - 35’13), occupe dans le schéma triangulaire la place du «grammairien» dont parle Bourdieu (1982, p. 52), le maître de la langue institutionnelle. Il serait donc en position, linguistiquement parlant, d'arbitre, en dépit de son origine sociale inférieure. Sa langue est sur-articulée : c'est celle d'un maître dans sa classe. Il va peu à peu l'adapter à sa nouvelle fonction. C'est un des aspects, et non le moindre, de la performance de Louis Jouvet.

Mais dans cette scène de cinéma, les critères classificatoires ne sont pas que linguistiques. Les mouvements, le jeu, les regards soulignés par les cadrages ont une fonction essentielle.

Bourdieu signale ces éléments infralinguistiques comme décisifs dans le processus d' «intimidation» :

tout permet de supposer que les instructions les plus déterminantes pour la construction de l'habitus se transmettent sans passer par le langage et par la conscience [...] : la modalité des pratiques, les manières de regarder, de se tenir, de garder le silence ou même de parler [...] sont chargées d'injonctions qui ne sont si puissantes [...] que parce qu'elles sont silencieuses et insidieuses. (Bourdieu, 1982, p. 37)

Dans un film, ceci est vraiment affaire de mise en scène. Castel-Bénac examine l'allure de Topaze, qu'il fait tourner devant lui comme dans une cabine d'essayage ; lui fume vigoureusement tandis que le faible instituteur s'étrangle 
avec l'alcool; Suzy, elle, reste assise et tient un long fume-cigarette - alors que Castel-Bénac tète son mégot; Topaze jette un regard diagonal furtif sur le dos nu de Suzy. Tous ces jeux de scène n'ont pas qu'une valeur «psychologique», ils tressent des lignes de tension qui décrivent des rapports de force, des relations de pouvoir et de désir.

\section{Le renversement de situation}

Vient un moment où la dramaturgie du film donne au spectateur ce qu'il attend depuis le début : le dominé grignote des parcelles de pouvoir et finit par s’imposer aux autres protagonistes.

Dans ces passages (pièce III, 2, film 52'-54', et IV, 2, 72'-75'), le second surtout, Topaze devient maître de la langue et de la communication, non plus la langue scolaire et académique mais celle, froide (c'est dit dans une didascalie de la pièce) et pragmatique, de la politique.

Prêtons attention aux positions et aux attitudes des personnages dans la scène ; Topaze, cette fois, est assis derrière son bureau. Il porte monocle. Vêtu d'un costume foncé, il s'est rasé, ses cheveux sont plaqués. Sa métamorphose n'est pas seulement physique, mais aussi vocale. Castel-Bénac est contraint de rester debout. En costume gris, il va et vient et gesticule inutilement.

Dans ce diagramme, par conséquent, la langue (et la prononciation) de référence est un point aveugle autour duquel se placent : le locuteur «distingué» dont l'«articulation nonchalante » (Bourdieu, 1982, p. 55, n. 26) est le signe distinctif (Roger) ; la tentative d'imitation de celle-ci par Suzy, la parvenue ; l'hypercorrection «petite-bourgeoise » représentée par Castel-Bénac, l’homme politique se trouvant ainsi placé au milieu de la hiérarchie linguistique, si je peux dire; enfin, hors des cadres, l'observateur et le correcteur, Topaze, qui saura user du langage parlé en en modifiant les caractères pour asseoir sa conquête.

Louis Jouvet imprime au personnage les fondamentaux de son jeu d'acteur : modulation très particulière de ses phrases, voyelles traînantes, diction nasale qui lui est propre. Il occupe l'espace en projetant sa voix. Comme plus tard dans Knock, c'est autant l'interprète que le personnage qui s'impose à l'écran. Ce film - comme c'est parfois le cas du premier film d'un acteur raconte aussi l'ascension et l'affirmation de l'acteur Jouvet. Il éclôt lui aussi dans une position de pouvoir.

Le succès du film est dû en bonne partie à la simplicité de son mécanisme. Topaze, sorti en 1933, a pris avec le temps une valeur paradigmatique qui s'imposera dans le cinéma français quand il s'essaiera à représenter le jeu social et politique. Un film antérieur va nous permettre de montrer la récurrence de ces thématiques transférées du théâtre de boulevard au cinéma. 


\section{Le Roi : les accents marqueurs des régimes et des options politiques?}

\section{Antériorité de la pièce}

C'est une pièce de Robert de Flers, Gaston Armand de Caillavet et Emmanuel Arène qui date de 1908. On doit noter qu'elle est bien antérieure à Topaze, mais il faut penser que le succès de la pièce de Marcel Pagnol l'avait remise au goût du jour. On doit noter la quasi-contemporanéité des deux films (19301933) qui rend leur comparaison pertinente.

Louis Verneuil écrivit l'adaptation que tourna Pierre Colombier, un de ces «tâcherons » du cinéma populaire français aujourd'hui si décriés mais si populaires alors.

La politique est présente partout dans ce scénario, mais en filigrane. Le thème principal est la nécessité où se trouve un régime républicain de faire bon accueil à un monarque (débonnaire, il est vrai). Le prétexte en est futile : la visite du roi de Cerdagne en France provoque une certaine émotion qui remonte jusqu'au cabinet du ministre des Affaires étrangères, mais qui oppose surtout un nobliau de province et le député-maire de Chamarande, Bourdier, un républicain affiché, mieux : un « rouge». On est peut-être en Sologne, une histoire de chasse aux faisans est le déclencheur. Le fond du problème s'articule surtout autour du partage d'une maîtresse que le roi a jadis connue, que le marquis de Chamarande entretient et que Bourdier convoite. L'affaire provoque une crise ministérielle qu'il faut calmer car un traité commercial est en jeu. Finalement, afin de calmer Bourdier et permettre cette signature, lui est octroyé le ministère du Commerce.

En 1930, ces allusions, ces mœurs, ne devaient pas paraître très exotiques aux administrés de la Troisième République.

\section{Le jeu des langues et des accents}

Les acteurs du film ont, si j'ose dire, «l'accent des planches». On n'oublie jamais et on ne masque pas qu'il s'agit d'une pièce filmée, même s'il y a de timides « respirations» en extérieurs. Cet accent, c'est le français standard qu'a décrit Michel Chion. Mais c'est par défaut, et par démarcation, que l'accent s'inscrit dans le signifiant sonore dans Le Roi, et il concerne d'abord deux personnages qui ne sont français ni l'un ni l'autre et par lesquels il faut commencer. C'est donc la typisation par l'accent étranger qui s'impose en premier lieu.

L'accent «cerdan » de la demi-mondaine Thérèse Marnix (Elvire Popesco) et du roi (Victor Francen) n'a rien de catalan! Il est donné en fait comme générique d'un accent «étranger». Vaguement «Europe centrale», il se marque essentiellement par le roulement des « $R$ », le déplacement de l'accent tonique, l'ouverture des syllabes. On a fait remarquer que le cinéma parlant des débuts, et le 
cinéma français tout au long de son histoire, a préféré de manière générale les acteurs à accent étranger aux acteurs à accent régional (Masson, 1989, p.121124). Sur le plan linguistique, ce roi est caractérisé par une emphase maladroite attribuable autant au caractère «oriental » supposé du locuteur qu'à son manque d'expérience de la langue française.

Ciné-Miroir (n 603, 23 octobre 1936) propose un reportage sur le tournage d'une scène entre Victor Francen et Gaby Morlay (qui joue Marthe, midinette devenue la femme de l'industriel Bourdier chez lequel le roi est hébergé) et note ainsi le dialogue :

«Oh, parrdon! fait le roi, avec un fort accent d'Europe centrale, je suis confus...

- Vous, sire? À cette heure?

- Oui, je suis sorrrti de chez moi parce qu'il y avait le feu... et n'ayant pas envie de fairrre sommeil, j'ai eu velléité d'aller causer politique avec le président de la commission des Restrrrictions...

Ciné-Monde ( ${ }^{\circ} 414,24$ septembre 1936) y allait aussi de son reportage. On y lit cet extrait du texte du roi découvrant sa chambre : "Oh! Oh! dit-il avec un accent étrangeraussi prononcé qu'indéfinissable, exceptionnellement décorrratif! chevalerrresque!»

Le même Cinémonde (n 437,4 mars 1937) nous éclaire spécialement sur la réception des accents de ce film. La journaliste (Alice Tessier) réitère les remarques sur le "fort accent étranger», cette fois celui de Thérèse (Popesco), et décrit ainsi le roi : "grand, bien découplé, avec des yeux tendres et rieurs, il s'exprimait avec recherche et son fort accent cerdanais apportait un charme de plus à sa voix, qu'il avait fort belle».

Quant à Bourdier, "gros industriel, homme vulgaire», on lui impute un «fort accent méridional » alors qu'on serait bien en peine d'en entendre, dans ce film, la moindre inflexion dans la bouche de Raimu...

Cette double méprise de la critique démontre que les présupposés formés par ce qu'on sait de l'acteur (Raimu) ou ce que l'on a retenu de l'intrigue (le roi de Cerdagne) font écran à la perception des accents, lesquels sont en outre, dans le cas précis, des accents fabriqués pour l'interprétation.

Reportons-nous pour un instant à ce que disent les linguistes de ce phénomène de l'identification des accents étrangers et régionaux par les auditeurs. Les spectateurs de cinéma en étant aussi, ce qui est dit ci-après leur est applicable.

«Les prototypes représentent des connaissances partagées qui, sans être universelles, sont utiles comme points de repère pour toute évaluation, pour faire des prédictions, pour organiser notre perception » (Boula de Mareüil et al., 2008, p. 151). Cette étude nous apprend les résultats d'une enquête qui prouve que le taux de réussite d'identification de l'origine linguistique des personnes est de $52 \%$ pour les accents étrangers (il est vrai qu'on n'a pas soumis au test 
un locuteur cerdan!) et de $43 \%$ pour les accents régionaux (ibid., p. 141 et 152). L'accent méridional est le mieux identifiable en français (p.153) mais avec des confusions sur les origines exactes. Il est noté aussi que le «R » roulé est l'un des éléments les plus discriminatoires, qui «peut colorer tout un énoncé et faire basculer la perception » (p.136).

Revenons au personnage de Bourdier. Le contrôle qu'il exerce sur son langage et son accent relèvent d'une double détermination, on l'a compris : le personnage de fiction, homme politique «local», doit parler la langue commune des hommes de pouvoir; l'acteur Raimu, pour s'insérer dans le «champ culturel» (pour reprendre le terme de J.-P. Esquenazi, 2004) qu'il visait, celui de la scène parisienne, s'efforce de gommer son accent en sur-corrigeant sa prononciation.

In fine, le maire Bourdier demeure «peuple» : il est d'ailleurs pris pour un secrétaire par l'espion du roi de Cerdagne. Mais l'acteur Raimu y ajoute sa marque, qui est le ton "déclamatoire», selon la terminologie de M. Chion (2003, p. 88) et sa gestuelle : il occupe l'espace, lance ses bras, secoue la tête de haut en bas avec vigueur. Les ruptures de ton font partie de sa panoplie, qu'il a imposée à tous ses réalisateurs. Ses scènes de colère en sont la traduction la plus fameuse (Raimu, comme Gabin, en a toujours au moins une par film). Le spectateur est libre d'y reconnaître des traits identitaires de l'homme du Midi, voire du méridional, quitte à prendre l'image qu'on lui tend pour un miroir où il voit son propre reflet.

Face à Bourdier, le marquis de Chamarande (André Lefaur, qui, détail curieux, avait été le créateur du rôle de Topaze sur scène), pratique à peu près la même langue, mais avec plus de «naturel» car elle est innée chez lui. Cela produit un effet de connivence, que renforce le fait qu'ils se sont succédé à la mairie, en dépit de leurs écarts d'opinion.

Marthe, le personnage que joue Gaby Morlay, se place à un rang inférieur. C'est une "ancienne arpète» (selon la critique de Cinémonde), une fille du peuple, une Parigote. Elle minaude, fait la folle, chante... On la voit même brièvement une fois imiter (mal) l'accent du Midi. Ce détail apporte la preuve que l'accent régional ne s'inscrit dans ce film que par défaut : c'est l'absent autour duquel en réalité tout s'organise. Le notable comme l'élu sont ceux qui s'en sont détachés.

On doit se garder de l'objection facile que ces films ne seraient que du théâtre filmé, car justement, cette considération est, elle aussi, porteuse de sens. La politique, c'est de la représentation. Sur l'écran, elle est au second degré. Martin Goutte a noté que c'est surtout le théâtre qui a été le modèle du jeu politique, comme il apparaît dans le langage commun (Gerstenkorn, Goutte, 2012, introduction). Si c'est aujourd'hui le petit écran qui est le lieu du «spectacle» politique, il est clair néanmoins que dans les années trente, le cinéma pouvait tenir ce rôle. Mais il l'a fait sur le registre de la caricature, 
mode d'expression qui est une façon médiatisée de saisir quelque chose du réel qu'il représente. Raimu parle à l'écran comme le faisaient les politiques sur les scènes de son temps; mais rien n'empêche de penser que les hommes politiques d'alors (et d'après) aient en retour emprunté des procédés à Raimu ${ }^{16}$. Quant à l'accent, nous avons vu qu'il est présent en creux. Contrairement à ce qu'écrivait André Bazin dans un article fameux ("le cas Pagnol », Bazin, 1959, II, p. 119-125), il n'est pas « consubstantiel au personnage» ou, plus exactement, cela, qui est vrai pour les films « méridionaux» de Pagnol, ne l'est plus pour les films à ambiance «politique ». C'est plutôt, dans les cas que nous avons examinés, un repoussoir latent que l'on a remplacé par une élocution apprise et affectée. Une superstructure, si l'on peut encore oser le terme... qui sert avant tout à se démarquer du parler ordinaire et à poser une situation de pouvoir ${ }^{17}$.

\section{Références}

AMY DE LA BRETÈQUe François, 1999, «Christian-Jaque», 1895, n²8, J. A. Gili et J. Lourcelles éd., p. 178-180.

AUMONT Jacques, MARIE Michel, 2001, «Fiction», Dictionnaire théorique et critique $d u$ cinéma, Paris, Nathan, p. 80.

Bazın André, 1959 [1950], « Le Cas Pagnol », Qu'est-ce que le cinéma, Paris, Le Cerf, t. II, p. $119-125$.

BeYLIE Claude, 1974, Marcel Pagnol, Paris, Seghers, coll. Cinéma d'aujourd'hui.

BILlARd Pierre, 1995, L’Âge classique du cinéma français. Du cinéma parlant à la Nouvelle Vague, Paris, Flammarion.

Boula de mareüll Philippe, Vieru-dimulescu Basil, Woehrling Cécile, 2008, «Accents étrangers et régionaux en français. Caractérisation et identification», Traitement automatique des langues, vol.49, nº3, p.135-163, en ligne : 〈http:// www.atala.org/IMG/pdf/TAL-2008-49-3-05-Boula.pdf> (consulté 6 juin 2016).

BouRdieu Pierre, 1982, Ce que parlerveut dire. L'économie des échanges linguistiques, Paris, Fayard.

CHION Michel, 1981, «L'homme politique dans le cinéma français», dossier de documentation du colloque de l'Institut Jean-Vigo, Perpignan.

- 2003, Un art sonore, le cinéma. Histoire, esthétique, poétique, Paris, Cahiers du cinéma.

CHIRAT Raymond, 1975, Catalogue des films français de long-métrage, films sonores de fiction 1929-1939, Bruxelles, Cinémathèque royale de Belgique.

16. Pour exemple Le Président, film d’Yves Jeuland consacré à Georges Frêche, président défunt de la Région Languedoc Roussillon (2010); voir l'article de Martin Goutte dans Cinémas en campagne, p. 44 et s.

17. Je remercie Maryse Beaute (service technique, université Paul-Valéry), Isabelle Debien (médiathèque d'agglomération de Montpellier) et Jacques Verdier (Cinémathèque eurorégionale de Perpignan, Institut Jean-Vigo). 
ESQUENAZI Jean-Pierre, 2004, Godard et la société française des années 1960, Paris, Armand Colin.

Gersten Korn Jacques, Goutte Martin, 2012, Cinémas en campagne. De la chronique électorale à la fiction politique, Lyon, Fage.

JeAnColas Jean-Pierre, 2005, Le cinéma des Français. 15 ans d'années trente (19291944), Paris, Nouveau Monde.

MASSON Alain, 1989, L'image et la parole. L'avènement du cinéma parlant, Paris, La Différence.

NACACHE Jacqueline, 2003, L'acteur de cinéma, Paris, Nathan.

Odin Roger, 2000, De la fiction, Bruxelles, De Boeck.

PAgnol Marcel, 1995 [1964], 1. La Cinématurgie de Paris [Les Cahiers du Film, décembre 1933 - mars 1934], Euvres complètes, t. III, Paris, Éd. de Fallois; 2. Fuvres complètes, t. I, Théâtre, Paris, Éd. de Fallois.

Peyrusse Claudette, 1986, Le cinéma méridional. Le Midi dans le cinéma français, 1929-1944, Toulouse, Eché.

Pithon Rémy, 1977, "Le Père Lampion », Les Cahiers de la Cinémathèque, n²3-24, p. 78-83.

PoMPA Dany, 1986, Marcel Pagnol, Paris, Henri Veyrier / Artefac.

SAdoul George, 1951, "Sur Topaze », L’Écran français, nº 291.

SMADJA David, 2010, "Cinématographie du politique ou l'écriture du politique comme image-mouvement», Raisons politiques. Études de pensée politique, n³8, p.5-15. 
